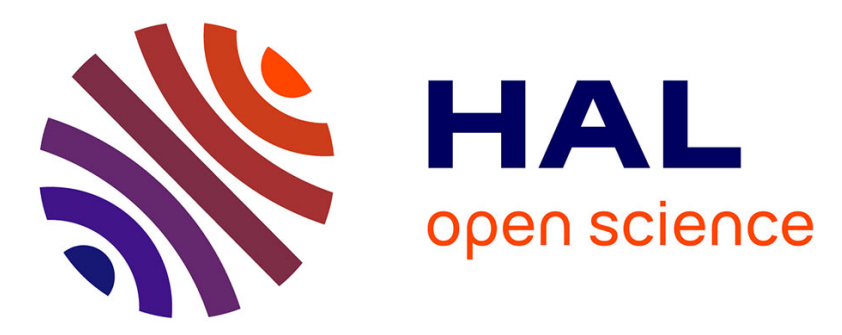

\title{
Restricted scaling range models for turbulent velocity and scalar energy transfers in decaying turbulence
}

\author{
F. Thiesset, R.A. Antonia, L. Danaila
}

\section{To cite this version:}

F. Thiesset, R.A. Antonia, L. Danaila. Restricted scaling range models for turbulent velocity and scalar energy transfers in decaying turbulence. Journal of Turbulence, 2013, 14 (3), pp.25 - 41. 10.1080/14685248.2013.803556 . hal-01660252

\section{HAL Id: hal-01660252 https://hal.science/hal-01660252}

Submitted on 28 Mar 2019

HAL is a multi-disciplinary open access archive for the deposit and dissemination of scientific research documents, whether they are published or not. The documents may come from teaching and research institutions in France or abroad, or from public or private research centers.
L'archive ouverte pluridisciplinaire HAL, est destinée au dépôt et à la diffusion de documents scientifiques de niveau recherche, publiés ou non, émanant des établissements d'enseignement et de recherche français ou étrangers, des laboratoires publics ou privés. 
Journal of Turbulence

Vol. 00, No. 00, 2011, 1-17

\title{
Restricted Scaling Range models for turbulent velocity and scalar energy transfers in decaying turbulence
}

\author{
F. Thiesset ${ }^{a, b *}$, R.A. Antonia ${ }^{a}$ and L. Danaila ${ }^{b}$ \\ ${ }^{a}$ School of Engineering, University of Newcastle, NSW 2308 Callaghan, Australia; \\ ${ }^{\mathrm{b}}$ CORIA, UMR 6614, Avenue de l'Université, BP 12, 76801 Saint Etienne du Rouvray, \\ France \\ (v3.2 released February 2009)

\begin{abstract}
The effect of finite Reynolds numbers and/or internal intermittency on the total kinetic energy and scalar energy transfers is examined in detail. For this purpose, two distinct models for velocity and scalar energy transfer are proposed in the specific context of freely decaying isotropic turbulence. The first one extends the already existing dynamical models (hereafter DYM, i.e. based on transport equations originated in Navier-Stokes and advection-diffusion transport equations). The second one relies on the characteristic time of the strain at a specific scale (hereafter SBM). Both models account for the Reynolds number dependence of the scaling exponent of the second-order structure functions, over a range of scales where such exponents may be defined, i.e. a Restricted Scaling Range (RSR). Therefore, the models developed aim at reproducing the energy transfer over the RSR. The predicted energy transfer is very sensible to variations of the scaling exponent, especially at low Reynolds numbers. The approach towards the asymptotic $4 / 3$ law is closely reproduced by the two models. The dynamical model reproduces the experimental results accurately especially in the vicinity of the Taylor microscale, whilst the SBM agrees almost perfectly with measurements at nearly all scales.
\end{abstract}

Keywords: Energy transfer, Finite Reynolds number, decaying isotropic turbulence

1. Introduction

2. Restricted scaling range models

2.1. The starting point. General equations

2.2. Dynamical Restricted scaling range models (DYM)

2.3. Strain-based models (SBM)

3. Validity of the models

3.1. Implementation

3.2. Comparison with an existing model

3.3. Comparison with experiments

3.3.1. Experimental details

3.3.2. Results

4. Summary

5. Acknowledgements

6. References

*Corresponding author. Email: fabien.thiesset@coria.fr

ISSN: 1468-5248 (online only)

(C) 2011 Taylor \& Francis

DOI: $10.1080 / 14685248$.YYYYxxxxxx

http://www.tandfonline.com 


\section{Introduction}

Starting with the Kármán-Howarth equation [1], Kolmogorov [2] derived a dynamical equation relating second- and third-order structure functions

$$
6 \nu \frac{\partial \overline{(\Delta u)^{2}}}{\partial r}-\overline{(\Delta u)^{3}}=\frac{4}{5} \bar{\epsilon} r
$$

In Eq. (1), $\nu$ denotes the kinematic viscosity, $\bar{\epsilon}$ is the mean energy dissipation rate, and $\Delta u(r)=u(x+r)-u(x)$ is the longitudinal velocity difference between two points separated by a distance $r$ in the streamwise direction. Hereafter, (.) denotes time averaging. For sufficiently high Reynolds numbers, an inertial range is established, within which the viscous term is negligible and the four-fifths law ensues:

$$
-\overline{(\Delta u)^{3}}=\frac{4}{5} \bar{\epsilon} r
$$

This exact result has received significant attention in the last half century either from theoretical or experimental perspectives. A similar equation was obtained by Yaglom [3] for a passive scalar such as the temperature fluctuation $\theta$ convected in a turbulent flow

$$
2 \kappa \frac{\partial \overline{(\Delta \theta)^{2}}}{\partial r}-\overline{\Delta u(\Delta \theta)^{2}}=\frac{4}{3} \overline{\epsilon_{\theta}} r .
$$

In Eq. (3), $\kappa$ is the thermal diffusivity and $\overline{\epsilon_{\theta}}$ is the mean dissipation rate of the scalar variance. In the inertial-convective range, the four-thirds law holds

$$
-\overline{\Delta u(\Delta \theta)^{2}}=\frac{4}{3} \overline{\epsilon_{\theta}} r .
$$

The four-fifths and four-thirds laws are exact results for fully developed, homogeneous and isotropic turbulence. There is consensus among the turbulence community that if a theory were to emerge, it must either recover these exact laws, or explicitly violate one of the hypothesis invoked in their derivations. In most laboratory experiments, the assumption that the Reynolds number is large is generally violated. Consequently, some additional terms reflecting the energy contribution of the largest scales must be retained in the energy budget equations.

In the last ten years, there has been a renewed interest in deriving such kinds of 'exact' relations [4-6] for low/moderate Reynolds number flows. It has been shown that the Kolmogorov four-fifths law is approached asymptotically [7-17]. Altogether, these studies have revealed that a Reynolds number $R_{\lambda}$ of order of $10^{3}$ in forced turbulence and $10^{5}$ in decaying turbulence must be reached for the four-fifths law to be nearly verified. $\left(R_{\lambda}=\sqrt{\overline{u^{2}}} \lambda / \nu\right.$ is the Reynolds number based on the Taylor microscale $\lambda=\sqrt{15 \nu \overline{u^{2}} / \bar{\epsilon}}$. For lower Reynolds numbers, the effect of the non-universal largest scales cannot be neglected so that deriving simple and general models of the energy transfer appears to be a challenging task.

There exist (at least) two classes of models for energy transfer: i) dynamical models and ii) phenomenological models. In the following, we briefly describe the most representative models of each class.

- Dynamical models (hereafter DYM). The starting point in developing such dynamical models is the transport equations for kinetic energy and the scalar vari- 
ance, which account for finite Reynolds numbers (FRN) effect. Specifically, in temporally decaying turbulence, Eqs. (1) and (3) write

$$
\begin{array}{r}
-\overline{(\Delta u)^{3}}+6 \nu \frac{\partial \overline{(\Delta u)^{2}}}{\partial r}-\frac{3}{r^{5}} \int_{0}^{r} s^{4} \frac{\partial \overline{(\Delta u)^{2}}}{\partial t} d s=\frac{4}{5} \bar{\epsilon} r . \\
-\overline{\Delta u(\Delta \theta)^{2}}+2 \kappa \frac{\partial \overline{(\Delta \theta)^{2}}}{\partial r}-\frac{1}{r^{3}} \int_{0}^{r} s^{2} \frac{\partial \overline{(\Delta \theta)^{2}}}{\partial t} d s=\frac{4}{3} \overline{\epsilon_{\theta}} r .
\end{array}
$$

The last term on the left hand side of Eqs. (5) and (6) characterizes the decay of kinetic energy. As noted for example in Ref. [6], this mechanism predominates when the separation $r$ is large, i.e. when $r$ is of the order of the integral scale.

These equations have been validated in grid turbulence at low/moderate Reynolds numbers [4] and reflect the balance between the decay, the transfer (third-order structure functions) and the dissipation of energy. Hence, considering that in Eqs. $(5,6)$ the unknown is the third-order term, modelling the second-order structure functions leads to evaluations of the energy transfer terms. If models for second-order structure functions only hold in the RSR, then the resulting energy transfer is expected to be reasonably evaluated over the same range of scales. The interest in deriving simple models for the energy (and/or scalar variance) transfer is to assess the validity limits of asymptotic equations such as Eqs. (2) and (4).

Along this direction, one type of model is that of Lindborg [9] for decaying turbulence. Another is that of Moisy et al. [10] in forced turbulence (this case is not addressed here). Both models have the main advantage of providing simple and purely analytical relations characterizing the energy transfer as a function of the scale $r$ and the Reynolds number $R_{\lambda}$. Their analysis mainly relies on the second similarity hypothesis [18], which allows the following expression for the second-order structure functions to be written

$$
\overline{(\Delta u)^{2}}=C_{u}(\bar{\epsilon} r)^{2 / 3} .
$$

However, Mydlarski \& Warhaft [19, 20] showed that the inertial range scaling exponents of velocity and scalar spectra asymptote towards $5 / 3$ as the Reynolds number increases. The same trend was revealed in physical space by Refs. [21, 22] for the scaling exponents of velocity and scalar second-order structure functions. On the other hand at high Reynolds numbers, the scaling exponent of structure functions $\zeta_{u}$ is generally larger than the asymptotic value of $2 / 3$. This 'anomalous' scaling has been extensively investigated and is commonly attributed to the effect of internal intermittency of inertial range fluctuations [23-25]. In the light of the ground-breaking work of Refs. [7, 8] and subsequent studies [9-17], it is important to mention that the internal intermittency phenomena cannot be unambiguously dissociated from FRN effects before a Reynolds number $R_{\lambda}$ of $5.10^{5}$ is reached. However, both phenomena lead to the same consequence, that is $\zeta_{u} \neq 2 / 3$ and the resulting Kolmogorov 'constant' $C_{u} \neq 2$.

In other words, the analysis of Refs. [9] and [10] emphasizes $R_{\lambda}$-variations of third-order structure functions without accounting for the $R_{\lambda}$-dependence of second-order structure functions. Whereas this approach is correct for sufficiently large Reynolds numbers, there appears to be a major incompatibility between different treatments of the second- and third-order terms. In addition, by virtue of the second similarity hypothesis (an inertial range law), these types of models are expected to approximate the energy transfer merely in the intermediate subrange. The transfer mechanism is unlikely to apply for either the viscous range 
or for large-scales.

This latter issue was tackled by Antonia et al. [14] and Antonia \& Burattini [16], who used an analytical expression of the second-order structure function [26]. Modelling the second-order structure functions over the whole range of scales should result in accurate predictions of the energy transfer at any scale (small, intermediate and large). The approach of Refs. [14, 16] provides a relatively accurate prediction of the energy transfer in isotropic freely decaying turbulence. In Refs. $[14,16]$, the scaling exponent $\zeta_{u}$ is set equal to $2 / 3$. FRN effects are thus implicitly accounted for through the variation of the Kolmogorov constant $C_{u}$ (see their Fig. (3)a in [16]). However, as mentioned in Refs. [14, 16], their model does not contain any information about the initial conditions that also affect the scaling exponent [27].

- Phenomenological models.

One type of phenomenological model is that of Oberlack \& Peters [28], for which the third-order correlation is calculated on the basis of a turbulent eddy viscosity. Note however that their model involves a constant (called $k_{0}$ in their paper) the value of which is determined in the context of infinite Reynolds numbers. Nevertheless, this kind of closure appears to be appropriate even at moderate Reynolds numbers (see their Fig. (1)).

Recently, a new type of model was proposed by Danaila et al. [29] in spectral space and by Danaila et al. [30] in physical space. These authors pointed to the imminent role played by the turbulent strain in the cascade mechanism. The energy transfer is thus interpreted as the loss of energy over a characteristic time scale which is simply the inverse of the strain at a scale $r$. The main advantage of this type of model is that the third-order structure function appears as a known functional of second-order structure functions, and is not constrained a priori by the knowledge of the full energy budget equation. This model was invoked notably to assess the evolution of the scaling exponent of temperature structure functions with respect to that of the kinetic energy and the Reynolds number. Nevertheless, Refs. [29, 30] were concerned only with the scalar field, and the extension of such a model to the velocity field is not straightforward.

The present study aims at investigating the effect of FRN and/or internal intermittency on the total kinetic energy transfer (the transfer of $\overline{\Delta u_{i} \Delta u_{i}}$ where summation applies) and on the scalar energy transfer, in the specific context of freely decaying isotropic turbulence. More precisely, two major questions are raised by the present study. (i) How can the dependence of the structure function scaling exponents on $R_{\lambda}$ and type of flow be incorporated in the models? (ii) How do these variations impact on the predicted energy transfer?

For this purpose, we first broaden the analysis developed in [9] in a significant manner, by accounting for the dependency on $R_{\lambda}$ and type of flow of the scaling exponents and the resulting Kolmogorov constant $C_{u}$. A second achievement of our analysis is to introduce a model for the scalar energy transfer along the lines of Kang \& Meneveau [31]. This framework enables an assessment of the conditions needed for the scalar field to behave in a similar manner to the velocity field.

We then propose an original strain-based model (SBM) in similar fashion to Danaila et al. [30]. We derive an equation regarding the scalar energy transfer and extend the analysis of Ref. [30] to the total kinetic energy transfer. Particular attention is paid to the input parameters required for an accurate parametrization of the latter model.

Both models are compared to experiments in different decaying flows spanning a wide range of Reynolds numbers.

The Restricted Scaling Range models developed in this work are presented in sec- 
tion 2. The dynamical model is presented in 2.2, whereas the strain-based model is derived in section 2.3. The comparison with an already existing model is performed in 3.2, whereas the validation against experiments is tackled in section 3.3. Finally, conclusions are drawn in section 4 .

\section{Restricted scaling range models}

\subsection{The starting point. General equations}

The kinetic energy budget equation of locally homogeneous temporally decaying isotropic turbulence can be written as

$$
F_{q}=\frac{4}{3}-\frac{2 \nu}{\bar{\epsilon} r} \frac{\partial \overline{(\Delta q)^{2}}}{\partial r}+\frac{1}{\bar{\epsilon} r^{3}} \int_{0}^{r} s^{2} \frac{\partial \overline{(\Delta q)^{2}}}{\partial t} d s
$$

In Eq. (8), $F_{q}=-\overline{\Delta u(\Delta q)^{2}} / \bar{\epsilon} r$. The quantities $\overline{(\Delta q)^{2}}=\overline{\Delta u_{i} \Delta u_{i}}$ and $\overline{\Delta u(\Delta q)^{2}}=$ $\overline{\Delta u\left(\Delta u_{i} \Delta u_{i}\right)}$ (summation applies to repeated Roman subscripts) represent the turbulent kinetic energy and the kinetic energy transfer at a scale $r$ respectively.

Further, the scale-by-scale equation of a passive scalar quantity such as the temperature fluctuation $\theta$ convected in a freely decaying isotropic flow (Eq. (3.8) Ref. $[4])$ is

$$
F_{\theta}=\frac{4}{3}-\frac{2 \kappa}{\overline{\epsilon_{\theta}} r} \frac{\partial \overline{(\Delta \theta)^{2}}}{\partial r}+\frac{1}{\overline{\epsilon_{\theta}} r^{3}} \int_{0}^{r} s^{2} \frac{\partial \overline{(\Delta \theta)^{2}}}{\partial t} d s
$$

In Eq. (9), $F_{\theta}(r)=-\overline{\Delta u(\Delta \theta)^{2}} / \overline{\epsilon_{\theta}} r$. The quantities $\overline{(\Delta \theta)^{2}}$ and $\overline{\Delta u(\Delta \theta)^{2}}$ represent the scalar energy and scalar energy transfer at a scale $r$ respectively. Note the mathematical equivalence between Eq. (8) and Eq. (9). Replacing $(\Delta q)^{2}$ by $(\Delta \theta)^{2}$, $\epsilon$ by $\epsilon_{\theta}$ and $\nu$ by $\kappa$ yields an analogous equation. This analogy was first noticed by Ref. [32], although the latter authors did not account for the large scale term.

When the flow under investigation is spatially decaying (grid, wake, jet turbulence), the Taylor's hypothesis is generally invoked so that the temporal derivative is replaced by $\frac{\partial}{\partial t} \equiv \bar{U} \frac{\partial}{\partial x}$.

Eqs. (8) and (9) provide some insight into why $F_{q}$ and $F_{\theta}$ may deviate from $4 / 3$. Indeed, at low Reynolds numbers, the two last terms on the RHS of Eqs. (8) and (9) are not negligible even in the RSR (Restricted Scaling Range) and thus cannot be discarded. For the $4 / 3$ law to be valid, the separation between the scales at which energy is injected and the viscous scales must be sufficiently large for an inertial range to develop. The latter requirement will be verified in the context of very large Reynolds numbers. This statement is fairly qualitative and we next turn our attention to this issue from a quantitative perspective. We use Eqs. (8) and (9) together with expressions for the second-order structure functions which contain explicit information about the effect of the Reynolds number (for both the scaling exponent $\zeta_{\beta}$ and the constant $C_{\beta}$, hereafter $\beta$ stands for either $q$ or $\theta$ ). We therefore derive dynamical models for $F_{q}$ and $F_{\theta}$ which inherit the dependence on $R_{\lambda}$.

\subsection{Dynamical Restricted scaling range models (DYM)}

This class of models is called 'dynamical' because of their origins in the dynamical transport equations (Navier-Stokes and advection-diffusion equations). 
These models aim at describing the energy transfer over a limited range of scales. To account for variations of the inertial range scaling exponent, we use the modified similarity forms [33]

$$
\begin{aligned}
& \overline{(\Delta q)^{2}}=C_{q}(\bar{\epsilon})^{2 / 3} r^{\zeta_{q}} \eta^{2 / 3-\zeta_{q}}, \\
& \overline{(\Delta \theta)^{2}}=C_{\theta}\left(\overline{\epsilon_{\theta}}\right)(\bar{\epsilon})^{-1 / 3} r^{\zeta_{\theta}} \eta^{2 / 3-\zeta_{\theta}},
\end{aligned}
$$

where $\eta=\left(\nu^{3} / \bar{\epsilon}\right)^{1 / 4}$ is the Kolmogorov [18] length-scale, $\zeta_{\beta}$ is the scaling exponent of $\overline{(\Delta \beta)^{2}}$, and $C_{\beta}$ the modified Kolmogorov or Obukhov-Corrsin [34] constant. In this context, both $\zeta_{\beta}$ and $C_{\beta}$ are functions of $R_{\lambda}$.

Following Ref. [9], we match Eq. (10) with Eq. (8), and after using the $k-\bar{\epsilon}$ model, viz. $\bar{U} \frac{\partial \bar{\epsilon}}{\partial x}=-2 C_{\epsilon 2} \bar{\epsilon}^{2} / \overline{q^{2}}\left(\overline{q^{2}}=\overline{u_{i} u_{i}}\right.$ is twice the total kinetic energy $k$ ), we obtain

$$
F_{q}(r)=\frac{4}{3}-C_{q}\left(2 \zeta_{q} r^{* \zeta_{q}-2}+C_{\star} R_{\lambda}^{-1} r^{* \zeta_{q}}\right)
$$

where $r^{*}=r / \eta, C_{\star}=\sqrt{\frac{5}{3}} 4 C_{\epsilon 2} / 3\left(\zeta_{q}+3\right)$. In freely decaying turbulence $C_{\epsilon 2}=$ $1-1 / m_{q}$ where $m_{q}<0$ is the decay exponent of the total kinetic energy. In the derivation of (12), the dependence of $\eta$ on $t$ has been neglected. This can be easily justified noting that the decay exponent of $\bar{\epsilon} \propto t^{n-1}$. It follows that the Kolmogorov scale $\eta$ grows as $t^{(1-n) / 4}$, therefore $\eta$ varies at a much smaller rate than $\bar{\epsilon}$. In addition, since in Eq. (10), the power of $\bar{\epsilon}$ is $2 / 3$ whilst that of $\eta$ is $2 / 3-\zeta_{q}$ (which rapidly goes to small values at large Reynolds numbers), variations of $\eta$ with respect to $t$ can be confidently neglected. This was verified $a$ posteriori and differences were of about $7 \%$ at $R_{\lambda}=50$ and negligible for Reynolds numbers larger than 200 .

The peak value of $F_{q}$ is

$$
A_{q}=\frac{4}{3}-4 C_{q}\left[2\left(2-\zeta_{q}\right) C_{\star}^{-1} R_{\lambda}\right]^{\zeta_{q} / 2-1} .
$$

Obviously, the same procedure can be applied to the scalar field. The equivalent $k-\overline{\epsilon_{\theta}}$ model reads [31] $\bar{U} \frac{\partial \overline{\epsilon_{\theta}}}{\partial x}=-2 C_{\epsilon_{\theta} 2}(\bar{\epsilon})\left(\overline{\epsilon_{\theta}}\right) / \overline{q^{2}}$, where $C_{\epsilon_{\theta} 2}=C_{\epsilon 2}\left(-m_{q}+\right.$ 1) / $\left(-m_{\theta}+1\right)$ where $m_{\theta}<0$ is the decay exponent of the scalar variance. After matching the $\overline{q^{2}}-\overline{\epsilon_{\theta}}$ model with Eqs. (11) and (9), yields

$$
F_{\theta}(r)=\frac{4}{3}-C_{\theta}\left(2 \zeta_{\theta} \operatorname{Pr}^{-1} r^{* \zeta_{\theta}-2}+C_{\star \star} R_{\lambda}^{-1} r^{* \zeta_{\theta}}\right)
$$

where $C_{\star \star}=2 \sqrt{\frac{5}{3}}\left(C_{\epsilon_{\theta} 2}-C_{\epsilon 2} / 3\right) /\left(\zeta_{\theta}+3\right)$. Note that the relation provided by Ref. [31] (see their Eq. (18)) is recovered in the specific case $\zeta_{\theta}=2 / 3$. The maximum value of $F_{\theta}$ is

$$
A_{\theta}=\frac{4}{3}-4 C_{\theta} \operatorname{Pr}^{-\zeta_{\theta} / 2}\left[2\left(2-\zeta_{\theta}\right) C_{\star \star}^{-1} R_{\lambda}\right]^{\zeta_{\theta} / 2-1}
$$

One interesting consequence is that this framework allows us to assess the conditions for which the velocity and the scalar field behave similarly, i.e. $F_{q}(r)=F_{\theta}(r)$. 
After matching the order and the coefficients of the polynomial, we obtain

$$
\left\{\begin{aligned}
\zeta_{\theta} & =\zeta_{q} \\
C_{q} \operatorname{Pr} & =C_{\theta} \\
C_{q} C_{\star} & =C_{\theta} C_{\star \star \star} .
\end{aligned}\right.
$$

The analogy between scalar and velocity fields is expected to hold if all conditions are respected. In grid turbulence, the scalar is generally injected by heating the flow $(\operatorname{Pr} \approx 1)$ via a mandoline. In addition, $m_{q} \approx m_{\theta}[35,36]$, so that $C_{\epsilon 2} \approx C_{\epsilon_{\theta} 2}$, even though the relative distance between the mandoline and the grid most likely has an influence [37]. However, as in the spectral domain [20, 29, 36], it is generally observed that $\zeta_{q} \neq \zeta_{\theta}[22,30]$. This remark is thus incompatible with the scalarvelocity analogy. To summarize, the present framework provides possible insight into the different behaviour of the scalar and the velocity energy transfers. More precisely, under the assumption of the present model and especially the use of the $k-\bar{\epsilon}$ model, we show that a necessary condition for the velocity and scalar fields to behave in similar fashion is that the scaling exponents of velocity and temperature second-order structure functions must be equal.

As a conclusion for the DYM, the required input parameters are the following:

$-R_{\lambda}, \zeta_{q}, C_{q}$ and $m_{q}$ for the velocity field, and

$-R_{\lambda}, \zeta_{\theta}, C_{\theta}, m_{q}$ and $m_{\theta}$ for the scalar field.

\section{3. $\quad$ Strain-based models (SBM)}

Antonia et al. $[24,32]$ suggested that $\Delta u(\Delta \theta)^{2} / r$ can be interpreted as the dissipation of the quantity $(\Delta \theta)^{2}$ over a time-scale of order $\Delta u / r$. In order to generalize the latter interpretation, we develop a relation similar to that proposed by Ref. [30] (see their Eq. (19)), by conjecturing that

$$
\frac{\overline{\Delta u(\Delta \beta)^{2}}}{r}(r)=K_{\beta}(r) \frac{\overline{(\Delta \beta)^{2}}(r)}{\tau(r)}
$$

Eq. (17) simply signifies that the energy transfer process can be represented as the loss of a quantity $\overline{(\Delta \beta)^{2}}$ over a period $\tau(r)$ which needs to be specified. In the study of Ref. [30], this relation pertained only to the scalar field $\beta \equiv \theta$. By virtue of the analogy between Eq. (8) and Eq. (9), we suppose that this relation is also valid for the kinetic energy. Consequently, the quantity $\beta$ in Eq. (17) denotes either $q$ or $\theta$.

The main challenge resides in formulating an adequate definition for $\tau(r)$. The key point in Ref. [30] is to consider that the time scale $\tau(r)$ is related to the turbulent strain intensity $S(r)$ i.e. $\tau(r)=S(r)^{-1}$. More precisely, $S(r)$ must represent the strain acting at a separation $r$ due to all scales larger than $r$ [30]. The general and isotropic formulations of $S(r)$ were recently derived by Ref. [38] to describe the local anisotropy in the wakes generating by different obstacles. A general expression of the strain at a scale $r$ was obtained, viz.

$$
S(\vec{r})=\left(\frac{\partial^{2}}{\partial r_{j} \partial r_{j}} \overline{(\Delta q)^{2}}\right)^{1 / 2}
$$

If local isotropy holds, the Laplacian operator can be rewritten in spherical coor- 
dinates, so that

$$
S(r)=\left(\frac{1}{r} \frac{\partial}{\partial r} \overline{(\Delta q)^{2}}+\frac{1}{2} \frac{\partial^{2}}{\partial r^{2}} \overline{(\Delta q)^{2}}\right)^{1 / 2}
$$

The first term on the RHS of Eq. (19) was already used by Ref. [30], and further recovered analytically by Ref. [38]. At small scales (the range of scales of interest for modelling the energy transfer), the second term on the RHS is less important. Secondly, at (very) large scales, even though it is not negligible, this term can lead to some non-physical negative values of $S^{2}$. These values may be attributed to errors in evaluating second-order derivatives from measured second-order structure functions. Hereafter, the latter term will be neglected so that we assume

$$
\tau(r)=\left(\frac{1}{r} \frac{\partial}{\partial r} \overline{(\Delta q)^{2}}\right)^{-1 / 2} .
$$

This strain-based model which will be referred to as SBM, allows mixed thirdorder statistics to be computed knowing only second-order statistics. However, the function $K_{\beta}$ is a priori unknown and will receive some attention in the following.

In the restricted scaling range

$$
\begin{aligned}
& \overline{\Delta u(\Delta q)^{2}}=A_{q} \bar{\epsilon} r \\
& \overline{\Delta u(\Delta \theta)^{2}}=A_{\theta} \bar{\epsilon}_{\theta} r .
\end{aligned}
$$

Assuming that $\zeta_{\beta}, C_{\beta}$ and $A_{\beta}=\max \left(F_{\beta}\right)$ vary with $R_{\lambda}$, the function $K_{\beta}$ can be evaluated. Considering Eq. (17) together with the restricted scaling range relations Eqs. (10), (11), (21) and (22) leads to

$$
\begin{aligned}
& K_{q}(r)=\sqrt{\frac{A_{q}^{2}}{\zeta_{q} C_{q}^{3}}}\left(r^{*}\right)^{\left(1-3 \zeta_{q} / 2\right)}, \\
& K_{\theta}(r)=\sqrt{\frac{A_{\theta}^{2}}{\zeta_{q} C_{q} C_{\theta}^{2}}}\left(r^{*}\right)^{\left(1-\zeta_{\theta}-\zeta_{q} / 2\right)} .
\end{aligned}
$$

Note that when FRN and intermittency corrections are applied, $K_{\beta}$ is a function of the Reynolds number through $\zeta_{\beta}, C_{\beta}$. The damping function $K_{\beta}$ further depends on the separation $r$. The latter feature appears as a "scaling" correction so that $\overline{\Delta u(\Delta \beta)^{2}}$ scales as $r^{1}$, in accord with the $4 / 3$ law. Unfortunately, the unknown constant $A_{\beta}$ (the maximum value of the energy transfer) appears in each relation. This means that, strictly speaking, the SBM is not a closure equation since $F_{\beta}$ is not a known functional of $\overline{(\Delta \beta)^{2}}$. $A_{\beta}$ may be flow dependent and thus needs to be evaluated according to the specific case under consideration.

\section{Validity of the models}

We first compare the present two models with the model of Refs. [14, 16]. This choice was motivated by the strong experimental evidence in support of that model, especially at low Reynolds numbers. 


\subsection{Implementation}

We first detail the implementation of the model Refs. [14, 16] (as a reference), followed by the new models developed in this work.

- The procedure employed by Refs. $[14,16]$ is as follows. A full analytical expression of the second-order structure function [26] is first invoked:

$$
\overline{(\Delta u)^{* 2}}=\frac{r^{* 2}}{15} \frac{\left(1+r^{*} / L^{*}\right)^{(2 c-2)}}{\left(1+\alpha r^{* 2}\right)^{c}},
$$

where $L^{*}$ is a measure of the integral scale estimated as $L^{*}=C_{\epsilon} R_{\lambda}^{3 / 2} 15^{-3 / 4}$. References $[14,16]$ assumed that the normalized dissipation rate $C_{\epsilon}=1$. The parameter $\alpha=\left(15 C_{u}\right)^{-3 / 2}$ is interpreted as the cross-over between dissipative and inertial scales. It follows that $c=1-\zeta_{u} / 2$ where $\zeta_{u}$ is the scaling exponent of $\overline{(\Delta u)^{2}} \cdot \overline{(\Delta q)^{2}}$ is then calculated using the isotropic relation

$$
\overline{(\Delta q)^{2}}=3 \overline{(\Delta u)^{2}}+r \frac{\partial}{\partial r} \overline{(\Delta u)^{2}}
$$

Instead of using the $\overline{q^{2}}-\bar{\epsilon}$ model, Refs. [14, 16] use the equilibrium similarity assumption so that the scale-by-scale budget Eq. (8) can be rewritten as

$$
\begin{array}{rr}
g_{q}+2 f_{q}^{\prime}-\left(\frac{5 \Gamma_{q 1}}{m_{q}}-10 \Gamma_{q 2}\right) \widetilde{r}^{-2}=\frac{20}{3} \widetilde{r}, \\
f_{q}(\widetilde{r})=\frac{\overline{(\Delta q)^{2}}}{\bar{q}^{2}} & g_{q}(\widetilde{r})=-\overline{\Delta u(\Delta q)^{2}} \frac{3^{1 / 2} R_{\lambda}}{\left(\overline{q^{2}}\right)^{3 / 2}}, \\
\Gamma_{q 1}=\int_{0}^{\widetilde{r}} \widetilde{s}^{3} f_{q}^{\prime} d \widetilde{s} & \Gamma_{q 2}=\int_{0}^{\widetilde{r}} \widetilde{s}^{2} f_{q} d \widetilde{s} .
\end{array}
$$

where $\tilde{r}=r / \lambda$, the superscript ' denoting differentiation with respect to $\widetilde{r}$ and $s$ is a dummy variable. Therefore the transfer term $g_{q}$ can be computed by difference since all the other terms of Eq. (27) depend only on the normalized second-order structure function $f_{q}$. Concerning the latter model we will retain the parameters of Refs. $[14,16]$, i.e. $C_{u}=C_{u}^{\infty}=2$ and $\zeta_{u}=\zeta_{u}^{\infty}=2 / 3$ (the superscript $\infty$ denotes an infinite Reynolds number and zero intermittency).

- By contrast, when FRN and internal intermittency effects are accounted for in both the DYM and the SBM, the procedure is as follows. $(i) \overline{\left(\Delta u^{*}\right)^{2}}$ is calculated using Eq. (25) with $C_{u}=2$. The evolution of $\zeta_{u}$ with respect to $R_{\lambda}$ is mimicked by the following empirical relation[21]

$$
\zeta_{u}=\zeta_{u}^{\infty} \exp \left(-\mathcal{R} / R_{\lambda}\right)
$$

$\mathcal{R}=15$ is chosen in accord with Ref. [21]. We assign to $\zeta_{u}^{\infty}$ the non intermittent value of $2 / 3$. This means that only FRN corrections are considered for the moment. (ii) $\overline{\left(\Delta q^{*}\right)^{2}}$ is then evaluated from the isotropic relation Eq. (26). (iii) $C_{q}$ is updated using

$$
C_{q}\left(R_{\lambda}\right)=\max \left(\overline{\left(\Delta q^{*}\right)^{2}} / r^{*} \zeta_{q}\right)
$$

where $\zeta_{q}=\zeta_{u}$. (iv) In what concerns the SBM, the input constant $A_{q}$ appearing in the damping function $K_{q}$ is provided by Eq. (13). 


\subsection{Comparison with an existing model}

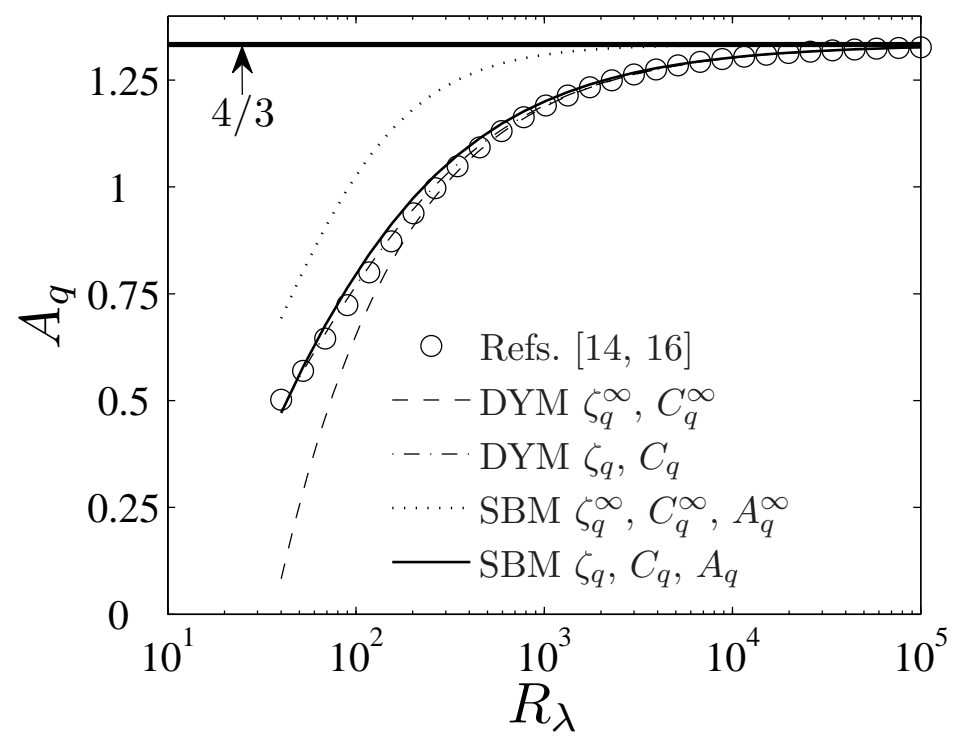

(a)

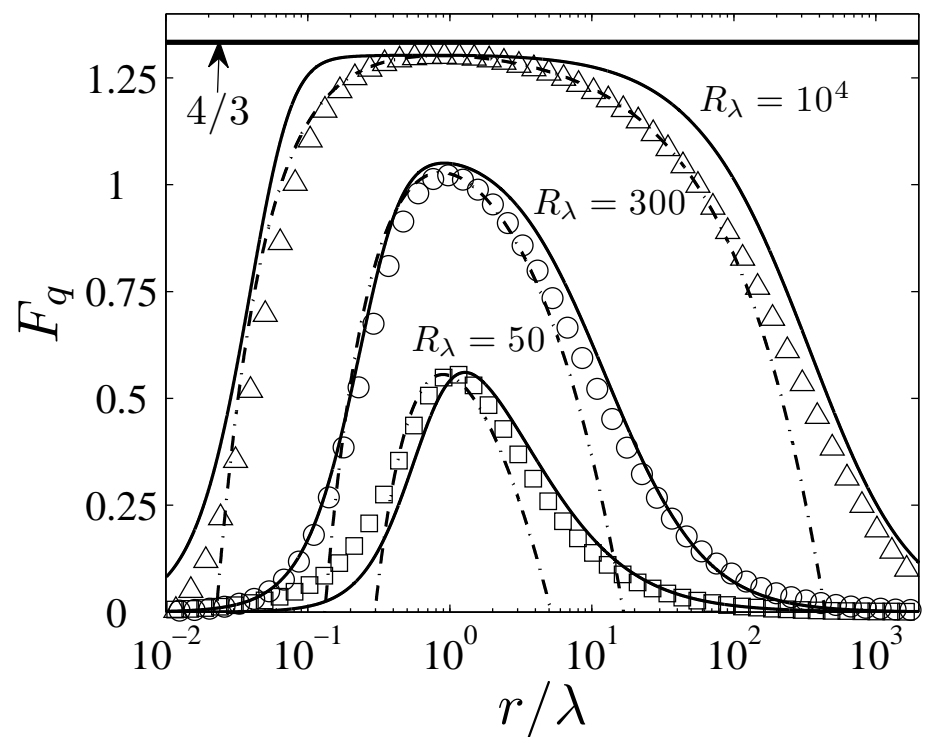

(b)

Figure 1. (a) Effect of FRN corrections on $A_{q}$ - - - - DYM without FRN corrections, - - — DYM with FRN corrections, $\cdots$... SBM without FRN corrections, — SBM with FRN corrections. Symbols refer to the model of Refs. [14, 16]. (b) $F_{q}$ inferred from the model of Refs. [14, 16] (symbols), DYM $(-\cdot-)$ and SBM (-). The Taylor microscale Reynolds number $R_{\lambda}=50,300$, and 10000. $m_{q}=-1$.

The first point to check is the ability of the present models to reproduce the approach towards the 4/3 law. In Fig. 1(a), we plot $A_{q}$ calculated with the DYM, as a function of the Reynolds number $R_{\lambda}$. First, when FRN effects are not taken into account $\left(C_{q}=C_{q}^{\infty}=22 / 3, \zeta_{q}=\zeta_{q}^{\infty}=2 / 3\right)$, the DYM leads to severely underestimated $A_{q}$ by comparison to the model of Refs. [14, 16], especially at low Reynolds numbers. When $R_{\lambda} \rightarrow 30, A_{q} \rightarrow 0$ which not surprisingly highlights the dominance of FRN effects. By taking into account the FRN corrections on $\zeta_{q}$, the DYM closely follows the expectation of Refs. $[14,16]$. Note that for $R_{\lambda} \approx 500$, the 
effect of FRN corrections is difficult to discern even though $A_{q}<A_{q}^{\infty}=4 / 3$.

In conclusion, the DYM can be reconciled with the model of Refs. [14, 16] when FRN effects are accounted for. Consequently, since $A_{q}$ is an input to the SBM, we expect the latter model to be accurately parametrized.

The maximum value of the energy flux is systematically overestimated by the SBM when the asymptotic values of $\zeta_{q}, C_{q}$ and $A_{q}$ are used (Fig. 1(a)). The value of the damping function $K_{q}$ is indeed non-physical since $C_{q}=22 / 3$ and $A_{q}=4 / 3$ are approached only at $R_{\lambda} \approx 10^{3}$ and $R_{\lambda} \approx 10^{5}$ respectively. The use of the 'real' values of $\zeta_{q}, C_{q}$ and $A_{q}$ leads to a strong reduction of errors. This is not surprising since the damping function ensures the matching of $A_{q}$ between DYM and SBM. In consequence, the SBM also faithfully reproduces the approach towards the asymptotic $4 / 3$ value.

The above discussion has focused on the maximum value of the energy transfer. We now turn our attention to the ability of the present models to reproduce the shape of the flux of energy at each scale. In the light of the previous remarks, only the models which account for FRN effects will be discussed below.

In Fig. 1(b), we compare results obtained from DYM and SBM together with the model of Refs. $[14,16]$. The tails of the transfer term at rather small and large scales are not reproduced by the DYM and a few negative (non physical) values are discernible. These negative values are due to the reliance of the DYM on the modified second similarity hypothesis which holds only in the inertial range. Nevertheless, in the vicinity of the Taylor microscale, the DYM follows the expected flux of energy.

On the other hand, the shape of the transfer term is well reproduced by the SBM at nearly all scales and not only in the RSR. This is surprising at first sight, since the model is calibrated through the damping function $K_{q}$ which relies on RSR relations. For large scales, the characteristic times grows faster than the damping function $K_{\beta}$, whereas the second order structure function tends to a constant. This results in rapidly decaying values of the energy flux for large scales. Consequently, the matching between the values of the model within the RSR and those at large scales is in quite good agreement with the predictions of the dynamical model of Refs. [14, 16].

We note however that at the largest Reynolds number $\left(R_{\lambda}=10^{4}\right)$, the plateau is wider compared to that of Refs. $[14,16]$. This emphasizes that the extent of the scaling range is larger for second-order statistics (input of the model) than for third-order moments. The widening of this range needs to be investigated further.

\subsection{Comparison with experiments}

In the previous section, for the purpose of illustrating the efficiency of the models, we decided to mimic the evolution of $\zeta_{q}$ by introducing the empirical relation Eq. (28). However, all the coefficients involved in the latter relation may depend on the type of flow $[21,22,27]$. This is not surprising since the principal issue of moderate Reynolds number flows is that the inertial range scales (if any) are unlikely to be universal. Hence, the two models need to be dynamically calibrated in the context of the flow under consideration, through the specific values of $\zeta_{\beta}$ and $C_{\beta}$. The next section addresses this topic by comparing the two models with experiments in different decaying flows.

\subsubsection{Experimental details}

Measurements were conducted in three types of decaying flows: slightly heated grid turbulence, cylinder wake and round jet flows. For the two latter flows, mea- 
surements only on the centreline or axis are considered to avoid any direct effect from the mean shear. These three different flows provide a sufficiently wide range of Reynolds numbers.

We acknowledge that, strictly, jet and wake flows are not freely decaying flows. For these flows, a production term must be retained in Eq. (8) even on the centerline[39]. However, in Ref. [39], the contribution of the production term is only $15 \%$ when $r \rightarrow \infty$ and is negligible for $r \leq 10 \lambda$ (see their Fig. 2). We conclude that the jet and wake flows remain good candidates for comparing the energy transfer with that inferred by the two present models, at least for $r \leq 10 \lambda$.

The grid turbulence experiments are described by Zhou et al.[35]. The wake flow is described in Antonia et al.[22,32], while the round jet is detailed in Antonia et $a l .[21]$ and Pearson[40]. The main flow parameters are listed in Table 1. The main features of the experimental facilities are briefly recalled below.

In all three flows, velocity and temperature fluctuations were measured using hot and cold wires respectively. Hot wires were etched from a Pt- $10 \%$ Rh Wollaston wire with a diameter of $2.5 \mu \mathrm{m}$ and a length to diameter ratio of nearly 200 . They were operated with in-house constant temperature anemometers at an overheat of 1.5. The cold wire diameter was $0.63 \mu \mathrm{m}$, operated with in-house constant current circuits. The ratio between the length and the diameter of the cold wire was about 1000. The magnitude of the current was $0.1 \mathrm{~mA}$.

For the grid turbulence, measurements were made on the centerline of a nonreturn wind tunnel of working section $350 \times 350 \mathrm{~mm}^{2}$. The biplane-grid mesh $M=$ $24.76 \mathrm{~mm}$ with a solidity of 0.35 , was composed of square rods $(4.76 \times 4.76 \mathrm{~mm})$. The mean velocity in the working section was $6.4 \mathrm{~ms}^{-1}$, and measurements were made in the range $20 \leq x / M \leq 80$. The flow was heated by use of a mandoline with a mesh size of $24.76 \mathrm{~mm}$ placed $1.5 \mathrm{M}$ downstream the grid. The temperature increase was about $2^{\circ} \mathrm{C}$. The probe consisted of two parallel hot wires straddling a X wire, and a pair of parallel cold wires. This allowed the measurement of one vorticity component $\omega_{i}$ and of the transverse temperature gradient. When placed in the $x-y$ plane, $u, v, \omega_{z}, \theta$ were measured while $u, w, \omega_{y}, \theta$ were obtained when the probe was in the $x-z$ plane.

\begin{tabular}{|c|c|c|c|c|c|c|c|c|}
\hline \multirow{2}{*}{ Flow } & $\bar{U}$ & $\overline{u^{2}}$ & $\overline{v^{2}}$ & $\overline{w^{2}}$ & \multirow{2}{*}{$\begin{array}{c}\overline{\theta^{2}} \\
10^{-2}\left({ }^{o} \mathrm{C}^{2}\right)\end{array}$} & \multirow{2}{*}{$\frac{\bar{\epsilon}}{10^{-2}\left(\mathrm{~m}^{2} \mathrm{~s}^{-3}\right)}$} & \multirow{2}{*}{$\frac{\overline{\epsilon_{\theta}}}{10^{-2}\left({ }^{\circ} \mathrm{C}^{2} \mathrm{~s}^{-1}\right)}$} & \multirow[t]{2}{*}{$R_{\lambda}$} \\
\hline & $\left(\mathrm{ms}^{-1}\right)$ & \multicolumn{3}{|c|}{$10^{-2}\left(\mathrm{~m}^{2} \mathrm{~s}^{-2}\right)$} & & & & \\
\hline Grid & 6.4 & 1.53 & 1.13 & 1.12 & 0.18 & 8.50 & 0.34 & 50 \\
\hline Wake & 12.9 & 66.9 & 58.7 & 60.7 & 1.67 & 892 & 11 & 230 \\
\hline Jet & 4.3 & 124.6 & 87.8 & - & - & 557 & - & 495 \\
\hline
\end{tabular}

Table 1. Relevant flow parameters. The mean dissipation rates $\bar{\epsilon}$ and $\overline{\epsilon_{\theta}}$ were inferred from Eqs. (30) and (31) respectively. These values are fully consistent with those obtained from the classical relations $\bar{\epsilon}=3 \nu \overline{\left(\partial u_{i} / \partial x\right)^{2}}$ and $\overline{\epsilon_{\theta}}=3 \kappa \overline{(\partial \theta / \partial x)^{2}}$. For grid turbulence, values are at $x=70 M$

The wake experiment was carried out in the same tunnel as that used for the grid turbulence experiments. The wake generator was a circular cylinder of diameter $d=25.4 \mathrm{~mm}$. The measurement location was at $x=70 d$. The aluminium cylinder was heated electrically so that the mean temperature on the centerline of the wake was about $0.43^{\circ} \mathrm{C}$ above ambient at $x / d=70$. The velocity fluctuations were measured with an $\mathrm{X}$-wire probe placed in the $x-y(u$ and $v)$ and in the $x-z$ 
plane $(u$ and $w)$ when the cylinder was not heated. When the cylinder was heated, $u$ and $\theta$ were measured simultaneously with a pair of parallel wires, one hot and one cold, separated in the transverse direction by $0.8 \mathrm{~mm}$.

The jet which issued from an axisymmetric nozzle of diameter $d=55 \mathrm{~mm}$, was supplied by a variable centrifugal blower. The measurement location was at $x / d=$ 40 , where the flow is approximately self-preserving. Only the velocity fluctuations $u$ and $v$ were measured by means of a $\mathrm{X}$ probe, and axisymmetry was invoked to obtain statistics related to $w$. The temperature was not measured in the latter flow. For more information about the jet, see Ref. [40].

\subsubsection{Results}

For low Reynolds number flows, it is difficult to discern a scaling range. Evaluating the scaling exponent from the local slope is thus tenuous and sometimes implies a degree of arbitrariness. As for Refs. [21, 22, 27, 41], we use a simple and direct method for computing the scaling exponents. It consists of fitting the experimental structure functions with the following scaling functions:

$$
\begin{aligned}
\overline{(\Delta q)^{2}} & =\frac{\bar{\epsilon}}{3 \nu} \frac{r^{2}}{\left[1+\left(r / r_{c_{q}}\right)^{2}\right]^{1-\zeta_{q} / 2}}, \\
\overline{(\Delta \theta)^{2}} & =\frac{\overline{\epsilon_{\theta}}}{3 \kappa} \frac{r^{2}}{\left[1+\left(r / r_{c_{\theta}}\right)^{2}\right]^{1-\zeta_{\theta} / 2}},
\end{aligned}
$$

where $r_{c_{\beta}}$ generally refers to the cross-over between viscous and inertial range scales. These two relations approximate the energy and temperature structure functions reliably from the smallest dissipative scales to inertial range scales. This "scaling function" method[42] was preferred to the Extended Self Similarity method (ESS) since the latter required a knowledge of $\overline{|\Delta u|^{3}}$ which is a priori unknown.

The least-squares fit is performed by use of the "nlinfit" function in the Matlab software. As in Refs. [21, 22, 27], the range of scales over which the least-squares fit is applied extends from the smallest separation to $r \approx \lambda$, viz. the separation at which $\overline{(\Delta u)^{3}} / r$ is maximum. Once the scaling exponent and the mean dissipation rate are known, the constants $C_{\beta}$ are calculated using either Eq. (10) or (11).

\begin{tabular}{cccccccc}
\hline \hline Flow & $R_{\lambda}$ & $\zeta_{q}$ & $C_{q}$ & $m_{q}$ & $\zeta_{\theta}$ & $C_{\theta}$ & $m_{\theta}$ \\
& & & & & & & \\
Grid & 50 & 0.29 & 16.57 & -1.36 & $0.51(0.48)$ & 6.15 & -1.33 \\
Wake & 230 & 0.55 & 12.96 & $-1^{\dagger}$ & $0.59(0.63)$ & 4.76 & $-1^{\dagger}$ \\
Jet & 495 & 0.70 & 6.96 & $-2^{\dagger}$ & - & - & - \\
& & & & & & & \\
\hline \hline
\end{tabular}

Table 2. Calculated values of $\zeta_{\beta}$ using the scaling functions method and resulting $C_{\beta}$. Values of $\zeta_{\theta}$ given in parentheses are inferred from $\zeta_{\theta}=\frac{1}{3}+\frac{\zeta_{q}}{2}[30]$. $\dagger$ In the wake and jet flows, measurements were made at only one streamwise location. Therefore, $m_{q}$ and $m_{\theta}$ cannot be evaluated. However, we will consider that self-similarity holds so that $m_{q}=m_{\theta}=-1$ in the wake flow and $m_{q}=-2$ in the jet flow. The latter result was confirmed from data of Ref. [43] obtained in the same jet flow.

Table 2 summarizes the different values of $\zeta_{\beta}$ and $C_{\beta}$ evaluated by the present scaling function method. One can note that $\zeta_{q}$ increases with the Reynolds number. This result is consistent with observations in the spectral space of Ref. [19] or in the physical space (Refs. [21, 22]). At $R_{\lambda}=495, \zeta_{q}$ is slightly larger than the K41 value of $2 / 3$. This reflects the effect of internal intermittency. The resulting Kolmogorov "constant" $C_{q}$ decreases with $R_{\lambda}$ similarly to what was observed by Ref. [19] in 
the spectral space. The value at $R_{\lambda}=495$ is slightly smaller than the expected non intermittent value $22 / 3$. We have to mention however, that this evolution with the Reynolds number is not meaningful since all these values may be also flow dependent.

One can further note that the scaling exponent of the scalar field is closer to the asymptotic non intermittent value. This result is fully compatible with the observed evolution of the scaling exponent in either the spectral space $[20,29,36]$ or the physical space[22]. In addition, measured values of $\zeta_{\theta}$ agree with the prediction of Ref. [30], i.e. $\zeta_{\theta}=\frac{1}{3}+\frac{\zeta_{q}}{2}$.

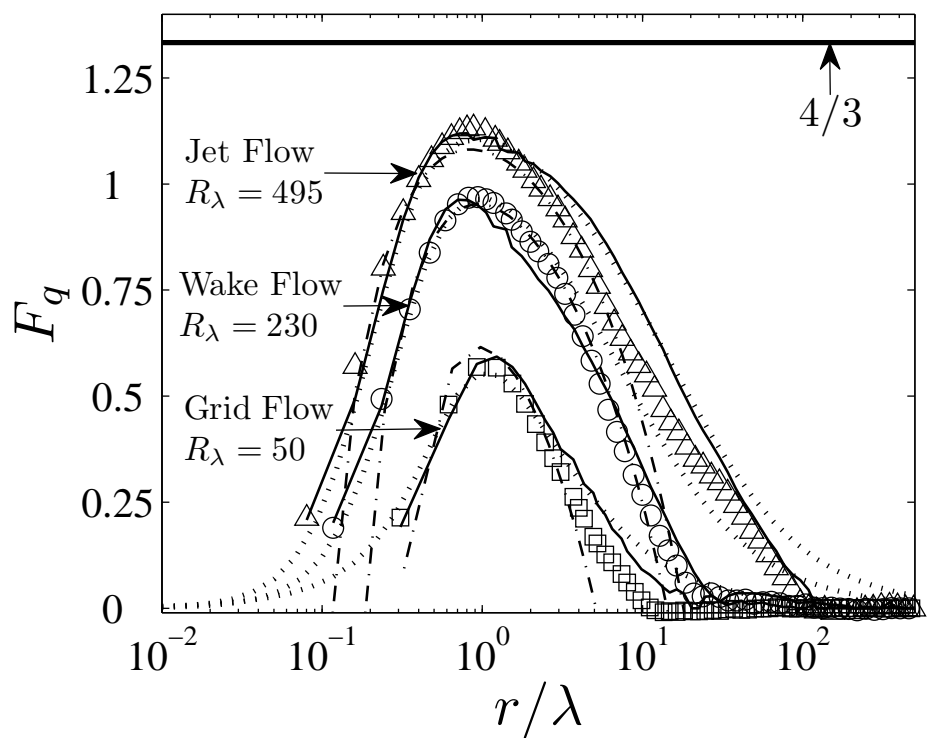

(a)

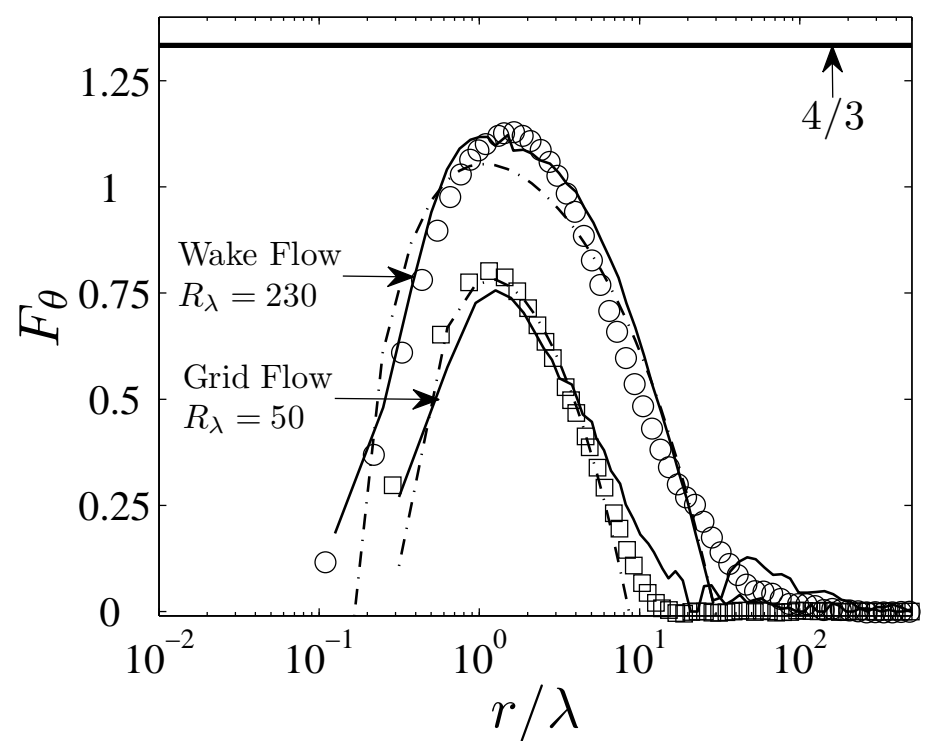

(b)

Figure 2. (a) Normalized turbulent energy transfer $F_{q}$ measured in different turbulent flows. $\square$ Grid flow $R_{\lambda}=50, \bigcirc$ Wake flow $R_{\lambda}=230, \triangle$ Jet flow $R_{\lambda}=495$. Comparison with the present SBM $(-)$ and the DYM (- - - ). Also included for comparison the model of Refs. $[14,16](\cdots \cdots)$. (b) Normalized scalar energy transfer $F_{\theta}$ measured in different turbulent flows. $\square$ Grid flow $R_{\lambda}=50$, $\bigcirc$ Wake flow $R_{\lambda}=230$. Comparison with the present SBM $(-)$ and the DYM $(-\cdot-)$ 
The measured energy transfer $F_{q}$ is compared with that inferred from the DYM and SBM in Fig. 2(a) for the three different flows. As for Fig. 1(b), DYM predicts accurately the shape of the energy flux and the maximum value $A_{q}$ in the vicinity of the Taylor microscale. This bodes well for the SBM which can thus be properly parametrized. Indeed, the agreement between SBM and measurements is nearly perfect for almost all scales. The main conclusion here is that the model first proposed in Ref. [30], originally devoted to the scalar field, can be readily extended to the total kinetic energy. The condition for the latter model to reproduce faithfully the energy flux is that it must be dynamically calibrated with respect to the flow and the Reynolds number under consideration.

Let us now turn our attention to the scalar field. In Fig. 2(b) we represent the scalar energy transfer term $F_{\theta}$ measured in the grid and wake flows. Note that the shape and particularly the maximum value of $A_{\theta}$ are substantially different to those observed in Fig. 2(a). More precisely, $A_{\theta}$ is larger than $A_{q}$ irrespectively of the flow considered. This difference between $F_{q}$ and $F_{\theta}$ is attributed to the difference between $\zeta_{\theta}$ and $\zeta_{q}$, and is accurately reproduced by both the DYM and the SBM.

\section{Summary}

The effect of finite Reynolds numbers and/or internal intermittency on the total kinetic energy and scalar energy transfers is investigated in detail. For this purpose, two separate models are derived for both the total kinetic energy and the scalar energy transfer in freely decaying isotropic turbulence. Both models are parametrized using the modified similarity hypothesis to account for the dependence of $\zeta_{\beta}$ and $C_{\beta}$ on the Reynolds number and type of flow. The experimental evaluation of the flow-dependent scaling exponent $\zeta_{\beta}$ is made in situ using the scaling functions method[42].

The SBM is based on the characteristic time-scale of the strain at a specific scale $r$. When deviations of the scaling exponents from the asymptotic value are accounted for, the damping function $K_{\beta}$ appears to be a function of the scale $r$. We interpret this feature as a scaling correction for consistency with the $4 / 3$ law. The second model (DYM), is the generalization of the models of Refs. $[9,31]$ in which the variations of $\zeta_{\beta}$ are explicitly accounted for. Within this framework, the analogy between the velocity and the scalar fields is re-analysed. We show that a necessary condition for the scalar and velocity fields to behave similarly is that they must have the same scaling exponent. This requirement is not satisfied in the different flows investigated, which is consistent with the observed difference between the kinetic energy and scalar energy transfers. We have to mention that the real-space behavior of the transferred energy at a given scale is perfectly equivalent to that evaluated in spectral space through Lin's equation. Therefore, the same conclusions hold in spectral space.

$F_{q}$ provided by either the SBM or the DYM has also been compared with the model of Refs. $[14,16]$. We show that the effect of the scaling exponent on the energy transfer is drastic, especially at low Reynolds numbers. When FRN and/or internal intermittency effect are taken into account, the DYM is well adapted solely in the vicinity of the Taylor microscale whilst the SBM agrees almost perfectly with the model of Refs. $[14,16]$ at nearly all scales. For the latter model, the predicted plateau in the inertial range is wider than that observed with both the DYM and the model of Refs. [14, 16]. Possible improvements to SBM are left for future work.

Since the energy transfer appears to be intimately connected to the flowdependent parameters $\zeta_{\beta}, C_{\beta}$ and $m_{\beta}$, there is no reason to expect a single universal approach of $A_{\beta}$ towards the asymptote. In contrast, there is most likely a network 
of possible solutions, even for the specific case of isotropic decaying turbulence. This suggests a possible explanation for the scatter in the experimental and DNS data[16] due to the effect of initial conditions.

\section{5. acknowledgements}

The financial support of the 'Agence Nationale de la Recherche' (ANR), under the project 'ANISO', is gratefully acknowledged. RAA acknowledges the support of the Australian Research Council.

\section{References}

[1] T. Kármán, and L. Howarth, On the statistical theory of isotropic turbulence, Proc. Roy. Soc. Lond. A 164 (917) (1938), pp. 192-215.

[2] A. Kolmogorov, Dissipation of energy in the locally isotropic turbulence, Dokl. Akad. Nauk. SSSR 125 (1941), pp. 15-17.

[3] A.M. Yaglom, On the local structure of a temperature field in a turbulent flow, Dokl. Akad. Nauk SSSR 69 (1949), p. 743.

[4] L. Danaila, F. Anselmet, T. Zhou, and R.A. Antonia, A generalization of Yaglom's equations which accounts for the large-scale forcing in heated decaying turbulence, J. Fluid Mech. 391 (1999), pp. 359-372.

[5] R.J. Hill, Exact second-order structure-function relationships, J. Fluid Mech. 468 (2002), pp. 317-326.

[6] L. Danaila, F. Anselmet, and R.A. Antonia, An overview of the effect of large scale inhomogeneities on small-scale turbulence, Phys. Fluids 14 (2002), pp. 2475-2484.

[7] J. Qian, Inertial range and the finite Reynolds number effect of turbulence, Phys. Rev. E 55 (1997), pp. 337-342.

[8] J. Qian, Slow decay of the finite Reynolds number effect of turbulence, Phys. Rev. E 60 (1999), pp. 3409-3412.

[9] E. Lindborg, Correction to the four-fifths law due to variations of the dissipation, Phys. of Fluids 11 (1999), pp. 510-512.

[10] F. Moisy, P. Tabeling, and H. Willaime, Kolmogorov Equation in a Fully Developed Turbulence Experiment, Phys. Rev. Lett. 82 (1999), pp. 3994-3997.

[11] T. Zhou, R.A. Antonia, L. Danaila, and F. Anselmet, Approach to the four-fifths law for grid turbulence, J. Turbulence 1(5) (2000), pp. 1-12.

[12] T.S. Lundgren, Kolmogorov two-thirds law by matched asymptotic expansion, Phys. Fluids 14 (2002), p. 638 .

[13] T.S. Lundgren, Linearly forced isotropic turbulence, CTR Annual Research Briefs (2003), pp. $461-473$.

[14] R.A. Antonia, R.J. Smalley, T. Zhou, F. Anselmet, and L. Danaila, Similarity of energy structure functions in decaying homogeneous isotropic turbulence, J. Fluid Mech. 487 (2003), pp. 245-269.

[15] Y. Gagne, and B. Castaing, Reynolds dependence of third-order velocity structure functions, Phys. Fluids 16 (2004), pp. 482-485.

[16] R.A. Antonia, and P. Burattini, Approach to the 4/5 law in homogeneous isotropic turbulence, J. Fluid Mech. 550 (2006), pp. 175-184.

[17] J. Tchoufag, P. Sagaut, and C. Cambon, Spectral approach to finite Reynolds number effects on Kolmogorov's 4/5 law in isotropic turbulence, Phys. Fluids 24 (2012), p. 015107.

[18] A. Kolmogorov, The local structure of turbulence in incompressible viscous fluid for very large Reynolds numbers, Proc. USSR Ac. of Sci. 30 (1941), pp. 299-303.

[19] L. Mydlarski, and Z. Warhaft, On the onset of high-Reynolds-number grid-generated wind tunnel turbulence, J. Fluid Mech. 320 (1996), pp. 331-368.

[20] L. Mydlarski, and Z. Warhaft, Passive scalar statistics in high-Pclet-number grid turbulence, J. Fluid Mech. 358 (1998), pp. 135-175.

[21] R.A. Antonia, B.R. Pearson, and T. Zhou, Reynolds number dependence of second-order velocity structure functions, Phys. Fluids 12 (2000), pp. 3000-3006.

[22] R.A. Antonia, T. Zhou, and G. Xu, Second-order temperature and velocity structure functions: Reynolds number dependence, Phys. Fluids 12 (2000), pp. 1509-1517.

[23] F. Anselmet, Y. Gagne, E.J. Hopfinger, and R.A. Antonia, High-order velocity structure functions in turbulent shear flows, J. Fluid. Mech. 140 (1984), pp. 63-89.

[24] R.A. Antonia, E.J. Hopfinger, Y. Gagne, and F. Anselmet, Temperature structure functions in turbulent shear flows, Phys. Rev. A 30 (1984), pp. 2704-2707.

[25] R. Benzi, S. Ciliberto, R. Tripiccione, C. Baudet, F. Massaioli, and S. Succi, Extended self similarity in turbulent flows, Phys. Rev. E 48 (1993), pp. 29-32.

[26] S. Kurien, and K.R. Sreenivasan, Anisotropic scaling contributions to high-order structure functions in high-Reynolds-number turbulence., Phys. Rev. E 62 (2000), pp. 2206-2212.

[27] R.A. Antonia, and B.R. Pearson, Effect of initial conditions on the mean energy dissipation rate and the scaling exponent, Phys. Rev. E 62 (2000), pp. 8086-8090.

[28] M. Oberlack, and N. Peters, Closure of the two-point correlation equation in physical space as a basis for Reynolds stress models, Applied Scientific Research 51 (1993), p. 533538. 
[29] L. Danaila, and R.A. Antonia, Spectrum of a passive scalar in moderate Reynolds number homogeneous isotropic turbulence, Phys. Fluids 21 (2009), p. 111702.

[30] L. Danaila, R.A. Antonia, and P. Burattini, Comparison between kinetic energy and passive scalar energy transfer in locally homogeneous isotropic turbulence, Phys. D 241 (2012), pp. 224-231.

[31] H.S. Kang, and C. Meneveau, Universality of large eddy simulation model parameters across a turbulent wake behind a heated cylinder, J. Turbulence 3 (2002), pp. 1-27.

[32] R.A. Antonia, M. Ould-Rouis, F. Anselmet, and Y. Zhu, Analogy between predictions of Kolmogorov and Yaglom, J. Fluid Mech. 332 (1997), pp. 395-409.

[33] K.R. Sreenivasan, On local isotropy of passive scalar in turbulent shear flows, Proc. Roy. Soc. A 434 (1991), pp. 165-182.

[34] A.M. Obukhov, The structure of the temperature field in a turbulent flow., Izv. Akad. Nauk. SSSR 13 (1949), p. 58

[35] T. Zhou, R.A. Antonia, L. Danaila, and F. Anselmet, Transport equations for the mean energy and temperature dissipation rates in grid turbulence, Exp. Fluids 28 (2000), pp. 143-151.

[36] S.K. Lee, A. Benaissa, L. Djenidi, P. Lavoie, and R.A. Antonia, Scaling range of velocity and passive scalar spectra in grid turbulence, Phys. Fluids 24 (2012), p. 075101.

[37] Z. Warhaft, and J.L. Lumley, An experimental study of the decay of temperature in grid turbulence, J. Fluid Mech. 88 (1978), pp. 659-684.

[38] F. Thiesset, L. Danaila, and R.A. Antonia, Dynamical effect of the total strain induced by the coherent motion on local isotropy in a wake., J. Fluid Mech. 720 (2013), pp. 393-423.

[39] P. Burattini, R.A. Antonia, and L. Danaila, Scale-by-scale energy budget on the axis of a turbulent round jet., J. Turbulence 6 (2005), pp. 1-11.

$40]$ B. Pearson, Experiments on small-scale turbulence, University of Newcastle, NSW, Australia, 1999.

[41] G. Stolovitzky, K.R. Sreenivasan, and A. Juneja, Scaling functions and scaling exponents in turbulence, Phys. Rev. E 48 (1993), p. R3217.

[42] K.R. Sreenivasan, and R.A. Antonia, The phenomenology of small-scale turbulence, Ann. Rev. Fluid Mech. 29 (1997), pp. 435-472.

[43] P. Burattini, R.A. Antonia, and L. Danaila, Similarity in the far field of a turbulent round jet, Phys. Fluids 17 (2005), p. 025101. 